We appreciate the response to this publication feature and welcome all contributions. Contributions may be sent to José

A. Mascorro, our Technical Editor, at his e-mail address:

jmascor@tulane.edu. José may also be reached at the

Department of Structural and Cellular Biology, Tulane University Health Sciences Center, 1430 Tulane Ave., New Orleans, LA 70112 and Ph: (504) 584-2747 Fax: (504) 584-1687

\section{Removing Very Fine Wrinkles from Half-Micron Epoxy Sections}

\author{
Bev Maleeff \\ GlaxoSmithKline \\ beverly.e.maleeff@gsk.com
}

Very fine wrinkles can occur at the tissue-resin interface when cutting half-micron thick sections of epoxy-embedded mouse intestine. The following is a list of possible remedies for removing such wrinkles:

- Adding glycerol to the water in the knife boat

- Using sodium ethoxide (or methoxide) to etch the resin

- Using chloroform, xylene or trichloroethylene fumes to stretch the sections

- Floating sections on a drop of $10 \%$ acetone on a slide, then heating gently to evaporate the acetone

- Place sections on a drop of water on a slide and then quickly flip the slide over. Lay the slide across a petri dish half-filled with xylene or chloroform so that the drop and sections are suspended above the solvent. Cover the petri dish and glass slide with a large staining dish for 5-10 minutes, then apply heat until dry.

While all of the above remedies are somewhat effective, the best solution in our laboratory is as follows:

- Place sections in a drop of water on a slide and stretch with chloroform fumes by waving a chloroform-soaked cotton swab over the sections

- Use a heat pen very close to (but not actually touching) the sections to stretch the sections yet again

- Gently heat the slide on a hot plate set to the lowest possible setting

Follow any of the above steps with the counterstain of your choice. This is far from a comprehensive list of tricks to eliminate wrinkles from sections, but these are the ones that were tried in this particular case.

\section{Problems with Hydrophobicity of Coated Grids \\ Jeanette Killius \\ NEOU College of Medicine \\ jkillius@neoucom.edu}

Occasional problems occur when attempting to place thin sections on Formvar-coated grids. The sections will not adhere to the grid but instead float away or fold upon themselves. This hydrophobicity problem is due to a microscopic build-up of an oily film on the grid surface. Techniques such as pre-wetting the grids prior to use or zapping the grids with an anti-static gun are not foolproof answers. The film must be removed to make the grids wettable again.

The problem can be easily resolved by using a vacuum evaporator fitted with a glow discharge cleaner. Place all grids, formvar-coated side up, onto a plastic Petri dish lined with filter paper. Leave the lid off the dish and place the dish in the center of the bell jar. Insert the aluminum cleaning wire into the proper port and pump down the chamber using the roughing pump to about $100 \mu \mathrm{m}$ on the thermocouple. Next, turn the selector switch to glow cleaning and slowly turn up the rheostat to 50. A violet haze will form around the wire and begin etching away the oily film from the grids while putting down a layer of negatively-charged ions. Allow the grids to be exposed to the haze for 3-5 minutes, periodically introducing a small amount of air into the chamber by using the chamber vent toggle switch. The chamber pressure should be maintained around $100 \mu \mathrm{m}$ throughout the cleaning period. At the end of the exposure time, turn down the rheostat completely, close the roughing pump, vent the chamber, and remove the grids. Grids cleaned in this manner may be used for up? to one week.

\section{The Versatile Family of Epoxy Resins: Designing Embedding Media with Specific Viscosity Properties}

José A. Mascorro

Tulane University School of Medicine jmascor@tulane.edu

Epoxy resins have been used successfully by biological microscopists for many years for studying the basic fine structure of tissues. Embedding media formulated with these basic resins are known for their ability to preserve morphological detail, minimally extract cell components, infiltrate tissues completely, and polymerize uniformly without inducing shrinkage artifacts. These media consistently produce hardened tissue blocks with favorablem sectioning and staining qualities, and the individual sections appear well-protected and capable of withstanding the heat and vacuum of the electron microscope column. For many years, Epon 812 served as the hallmark of epoxy resin embedding procedures. But due to environmental concerns, this valuable and historical resin was removed" from the market and has not been available since the middle 1970 s. Other epoxy replacement resins have since become widely available and the microscopist today can choose from many products when planning the make-up of the embedding medium.

For many years, microscopists purchased an embedding media "kit" that always contained the resin Epon 812 coupled with the anhydrides dodecynyl succinic anhydride (DDSA) and nadic methyl anhydride (NMA) and the amine accelerator 2,4,6-Tri(dimethylamin omethyl)phenol (DMP-30). This pre-packaged kit produced good results, but the final medium exhibited a very high viscous character that could not be lowered, assuming the microscopist wished to do so, because of the inherent high viscosity of the individual components On the other hand, the replacement resins are highly versatile and can be very specifically "mixed-and-matched" with particular anhydrides and catalysts so as to produce for the microscopist a completed medium with the desired viscosity to suit the particular tissue under consideration (Table l). Epoxy resin media, however their final make-up may be designed, nevertheless are naturally viscous and could cause infiltration wave damage or incomplete impregnation. Presumably, the less viscous mixtures would lessen this possibility while still achieving the primary purpose of complete tissue infiltration subsequent to fina polymerization. This work categorizes individual epoxy resins as well as the anhydrides and catalysts into a low, intermediate, or high viscous range. The components then can be matched in order to design and produce a complete medium with a specified viscosity. The final viscous character of the medium can be determined, and performance characteristics such as infiltration, polymerization, sectioning, and staining evaluated. This "mix-and-match" technique yields excellent media and ensures that the microscopist will achieve predictable results that fit the demands of the tissue being studied. A series of reports, to appear in future issues, will list the various physica parameters, including flow rate, average flow rate, volume flow rate, and viscosity of the more commonly utilized epoxy resins, anhydrides, catalysts and completed mixtures.

Summary of Viscosities

\begin{tabular}{|c|c|c|}
\hline Low Viscosity: & Medium Viscosity: & High Viscosity: \\
\hline $\begin{array}{l}\frac{\text { Resin }}{\text { VCD }} \\
\text { Transmit } \\
\text { Embed } 812\end{array}$ & $\begin{array}{l}\text { Resin } \\
\text { Medcast } \\
\text { LX } 112 \\
\text { Polybed } 812\end{array}$ & $\begin{array}{l}\text { Resin } \\
\text { Scipoxy } 812 \\
\text { Eponate } 812 \\
\text { Epon } 812\end{array}$ \\
\hline$\frac{\text { Anhydride }}{\text { NSA }}$ & $\frac{\text { Anhydride }}{\text { NMA }}$ & $\frac{\text { Anhydride }}{\text { DDSA }}$ \\
\hline$\frac{\text { Catalyst }}{\text { BDMA }}$ & & $\frac{\text { Catalyst }}{\text { DMP-30 }}$ \\
\hline
\end{tabular}

